EPJ manuscript No.

(will be inserted by the editor)

\title{
Cotunneling through a quantum dot coupled to ferromagnetic leads with noncollinear magnetizations
}

\author{
Ireneusz Weymann ${ }^{1}$ and Józef Barnaś ${ }^{1,2}$ \\ 1 Department of Physics, Adam Mickiewicz University, 61-614 Poznań, Poland \\ 2 Institute of Molecular Physics, Polish Academy of Sciences, 60-179 Poznań, Poland \\ Received: date / Revised version: date
}

\begin{abstract}
Spin-dependent electronic transport through a quantum dot has been analyzed theoretically in the cotunneling regime by means of the second-order perturbation theory. The system is described by the impurity Anderson Hamiltonian with arbitrary Coulomb correlation parameter $U$. It is assumed that the dot level is intrinsically spin-split due to an effective molecular field exerted by a magnetic substrate. The dot is coupled to two ferromagnetic leads whose magnetic moments are noncollinear. The angular dependence of electric current, tunnel magnetoresistance, and differential conductance are presented and discussed. The evolution of a cotunneling gap with the angle between magnetic moments and with the splitting of the dot level is also demonstrated.
\end{abstract}

PACS. 72.25.Mk Spin transport through interfaces - 73.63.Kv Quantum dots - 85.75.-d Magnetoelectronics; spintronics: devices exploiting spin polarized transport or integrated magnetic fields -73.23 . Hk Coulomb blockade; single-electron tunneling

\section{Introduction}

The vast step forward in fabrication of tunnel junctions ' has enabled the implementation of extremely small metallic grains and semiconductor quantum dots coupled through ' tunnel barriers to external reservoirs [12] . Currently, transport through such ultra-small devices is being extensively 'studied because of many future application possibilities and, more importantly, because of beautiful physics that emerges in these nanoscale systems. In nanostructures not ' only the manipulation of a single electron charge is possible, but - when coupled to ferromagnetic leads - also the manipulation of a single electron spin. This is why those systems are considered to play an important role in spintronic devices.

An interesting feature of electronic transport through nanoscale systems coupled to ferromagnetic leads is the tunnel magnetoresistance (TMR). The TMR effect, although discovered already three decades ago in planar junctions [3, is still of current interest. It consists in a change of the system conductance when relative orientation of the magnetic moments of external leads switches from antiparallel to parallel alignment. In a general case, magnetic moments of the two electrodes can form an arbitrary angle (noncollinear configuration). Qualitatively different features of electron transport through nanosystems appear due to discrete charging effects, leading to Coulomb blockade and Coulomb oscillations of electric current. The interplay of charge and spin effects gives rise to new interesting phenomena, like for instance TMR oscillations with applied bias and gate voltages 456789 .

Theoretical considerations of electron tunneling through quantum dots attached to ferromagnetic leads have already been reported in a number of papers. Most of them, however, are restricted to spin-dependent transport in systems with collinear alignment of the electrodes' magnetizations. Basically, all transport regimes in such a geometry have already been addressed, including sequential (first order) tunneling 1011, cotunneling (second order) 12, resonant tunneling [1314, and Kondo regimes 1516. Spin polarized transport through quantum dots coupled to ferromagnetic electrodes with noncollinear magnetic moments is still not fully explored, although it has already been considered in a couple of papers [17/1819202122].

In this paper we address the problem of second-order (cotunneling) spin-dependent transport through quantum dots coupled to ferromagnetic leads with arbitrary configuration of the in-plane magnetic moments of external electrodes. The considerations are limited to single-level quantum dots. Moreover, the level of the corresponding isolated dot is assumed to be spin-split due to coupling between the dot and a magnetic substrate on which the dot is deposited. The corresponding splitting is assumed to be larger than the level width $\Gamma$ due to coupling of the dot to external leads.

The paper is organized as follows. The model and method are described in section II. Transport through an empty quantum dot in the cotunneling regime is described in 
Please give a shorter version with: lauthorrunning

section III, where also the relevant numerical results are presented and discussed. Section IV covers the problem of cotunneling through a singly occupied dot. Final conclusions are given in section $\mathrm{V}$.

\section{Description of the model and method}

The system considered in this paper consists of a singlelevel quantum dot coupled through tunnel barriers to two external ferromagnetic leads, whose magnetizations are oriented arbitrarily in the plane of the structure. The dot is assumed to be deposited on a ferromagnetic substrate which strongly interacts with the dot and leads to spinsplitting of the dot level. The splitting is assumed to be larger than the level splitting due to exchange interaction between the dot and electrodes. The exchange interaction results from tunneling processes and is of the first order in the coupling parameter $\Gamma$ [18 19]. When neglecting the exchange coupling between the dot and leads, one may assume that the level splitting is constant, i.e., independent of applied voltage.

Coupling of the dot to ferromagnetic substrate is described by an effective molecular field $\mathbf{B}_{s}$, lying in the plane of the structure. Thus, the magnetic moments of the electrodes and the molecular field are all in a common plane. The effective molecular field determines the global quantization axis for electron spin on the dot (axis $z$ antiparallel to the molecular field). In turn, the local quantization axis in the leads is determined by the corresponding local magnetization direction, and is parallel to the net spin of the lead (thus, being antiparallel to the local magnetic moment). Furthermore, we assume that the net spin of the left (right) lead forms an angle $\varphi_{\mathrm{L}}\left(\varphi_{\mathrm{R}}\right)$ with the global quantization axis as shown in Fig. 11 In order to distinguish between different quantization directions, the majority (minority) spins in the local reference systems are labelled with $\sigma=+(-)$, while spin projection on the global quantization axis is denoted as $\sigma=\uparrow(\downarrow)$ for spin-up (spin-down) electrons.

Hamiltonian of the system has the general form $H=$ $H_{\mathrm{L}}+H_{\mathrm{R}}+H_{\mathrm{D}}+H_{\mathrm{T}}$, where $H_{\mathrm{L}}$ and $H_{\mathrm{R}}$ describe the left and right leads as reservoirs of noninteracting quasiparticles, $H_{\mathrm{D}}$ is the dot Hamiltonian, and tunneling processes between the electrodes and dot are included in $H_{\mathrm{T}}$. The lead Hamiltonians are diagonal in the respective local coordinate systems, $H_{\alpha}=\sum_{\sigma=+,-} \sum_{k \in \alpha} \varepsilon_{\alpha k \sigma} a_{\alpha k \sigma}^{\dagger} a_{\alpha k \sigma}$ (for $\alpha=\mathrm{L}, \mathrm{R}$ ), with $\varepsilon_{\alpha k \sigma}$ being the energy of a single electron with wavevector $k$ and spin $\sigma$ in the lead $\alpha$, whereas $a_{\alpha k \sigma}^{\dagger}$ and $a_{\alpha k \sigma}$ denote the corresponding creation and annihilation operators.

The dot is described by the Anderson Hamiltonian, which in the global reference frame can be expressed as $H_{\mathrm{D}}=\sum_{\sigma=\uparrow, \downarrow} \varepsilon_{\sigma} d_{\sigma}^{\dagger} d_{\sigma}+U n_{\uparrow} n_{\downarrow}$, where $\varepsilon_{\sigma}$ is the energy of an electron with spin $\sigma, \varepsilon_{\sigma}=\varepsilon \mp g \mu_{B} B_{s}$, and $d_{\sigma}^{\dagger}\left(d_{\sigma}\right)$ creates (annihilates) a spin- $\sigma$ electron. Here, $g$ is the Lande factor and $\varepsilon$ is the dot level energy in the absence of magnetic electrodes and molecular field due to the substrate. The second term of the dot Hamiltonian describes

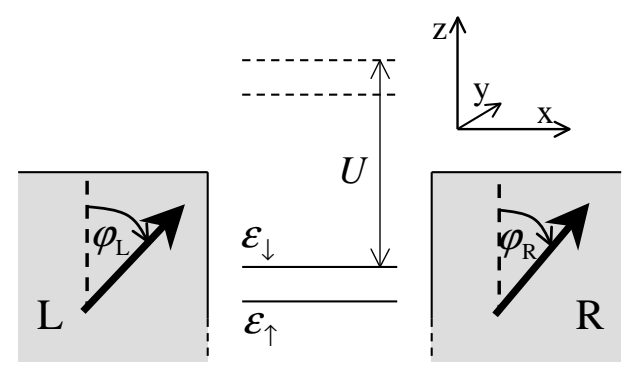

Fig. 1. The schematic of a magnetic quantum dot coupled to ferromagnetic leads. Classical spin of the lead $\alpha(\alpha=\mathrm{L}, \mathrm{R})$ can form an arbitrary angle $\varphi_{\alpha}$ with the dot spin quantization axis (axis $z$ ), as indicated.

Coulomb interaction of two electrons of opposite spins residing on the dot, with $U$ denoting the corresponding correlation energy. The tunnel Hamiltonian takes the form

$$
\begin{aligned}
H_{\mathrm{T}} & =\sum_{\alpha} \sum_{k \in \alpha}\left[\left(T_{\alpha k+} a_{\alpha k+}^{\dagger} \cos \frac{\varphi_{\alpha}}{2}-T_{\alpha k-} a_{\alpha k-}^{\dagger} \sin \frac{\varphi_{\alpha}}{2}\right) d_{\uparrow}\right. \\
& +\left(T_{\alpha k+} a_{\alpha k+}^{\dagger} \sin \frac{\varphi_{\alpha}}{2}+T_{\alpha k-} a_{\alpha k-}^{\dagger} \cos \frac{\varphi_{\alpha}}{2}\right) d_{\downarrow}+\text { h.c.)(1) }
\end{aligned}
$$

with $T_{\alpha k \sigma}$ denoting the tunnel matrix elements between the dot states and majority $(\sigma=+)$ or minority $(\sigma=-)$ electron states in the lead $\alpha$ when $\varphi_{\alpha}=0$.

Due to the coupling between dot and leads, the dot level acquires a finite width. When the magnetic moment of lead $\alpha$ and the molecular field acting on the dot are parallel, the corresponding contribution $\Gamma_{\alpha}^{\sigma}$ to the dot level width may be written as $\Gamma_{\alpha}^{\sigma}=2 \pi\left|T_{\alpha \sigma}\right|^{2} \rho_{\alpha \sigma}$, where $\rho_{\alpha \sigma}$ is the spin-dependent density of states for the majority $(\sigma=+)$ and minority $(\sigma=-)$ electrons in the lead $\alpha$. The parameters $\Gamma_{\alpha}^{\sigma}$ will be used in the following to parameterize strength of the coupling between the dot and lead $\alpha$. It is convenient to express the coupling parameters in terms of spin polarization defined as $P_{\alpha}=$ $\left(\Gamma_{\alpha}^{+}-\Gamma_{\alpha}^{-}\right) /\left(\Gamma_{\alpha}^{+}+\Gamma_{\alpha}^{-}\right)$. Thus, the coupling strength can be written as $\Gamma_{\alpha}^{ \pm}=\Gamma_{\alpha}\left(1 \pm P_{\alpha}\right)$, where $\Gamma_{\alpha}=\left(\Gamma_{\alpha}^{+}+\Gamma_{\alpha}^{-}\right) / 2$. In our considerations we assume $\Gamma_{\mathrm{L}}=\Gamma_{\mathrm{R}}=\Gamma / 2$.

In the case of ferromagnetic leads, the coupling of the spin-up dot level is different from the coupling of the spin-down level, which is due to different densities of states for spin-majority and spin-minority electron bands in the leads. This may result in the splitting of the dot level 1223 . Here, we assume that the dot level splitting, $\Delta=\varepsilon_{\downarrow}-\varepsilon_{\uparrow}$, due to the molecular field is larger than the coupling parameters, $\Delta \gg \Gamma_{\alpha}^{ \pm}$. In other words, we assume that the exchange interaction between the dot and magnetic leads is much smaller than the Zeeman energy due to the molecular field and can be neglected. An electron residing on the dot has then either spin up or down. Thus, the corresponding density matrix in the global quantization system is diagonal in the spin space 24]. In that case, only the second-order processes (with respect to the tunneling Hamiltonian) have to be taken into account in the Coulomb blockade regime. When the above condition is not fulfilled, the exchange interaction cannot be ignored 
and therefore the first-order processes, which are responsible for the exchange coupling (and do not contribute to charge transport), have to be considered.

In the Coulomb blockade regime, the energy needed to put an electron on the dot surpasses the energy provided by the transport voltage and the sequential tunneling is exponentially suppressed. The dot is then in a well defined charge state, and quantum charge fluctuations are suppressed. Although the energy conservation prohibits the first-order tunneling transitions, the current can still be mediated by higher-order tunneling processes involving correlated tunneling of two (cotunneling) or more electrons via intermediate virtual states 25.

The rate of electron cotunneling from a spin-majority state in the lead $\alpha$ to a spin-majority state in the lead $\beta$ can be determined using the second-order perturbation theory [2526], and is given by

$$
\gamma_{\alpha \beta}^{+\Rightarrow+}=\frac{2 \pi}{\hbar}\left|\sum_{v} \frac{\left\langle\Phi_{\alpha}^{+}\left|H_{\mathrm{T}}\right| \Phi_{v}\right\rangle\left\langle\Phi_{v}\left|H_{\mathrm{T}}\right| \Phi_{\beta}^{+}\right\rangle}{\varepsilon_{i}-\varepsilon_{v}}\right|^{2} \delta\left(\varepsilon_{i}-\varepsilon_{f}\right),
$$

where $\left|\Phi_{\alpha}^{+}\right\rangle$and $\left|\Phi_{\beta}^{+}\right\rangle$are the initial and final states of the system, whereas $\left|\Phi_{v}\right\rangle$ is a virtual state. The corresponding energies are denoted as $\varepsilon_{i}, \varepsilon_{f}$, and $\varepsilon_{v}$.

One can distinguish cotunneling processes that change the magnetic (and consequently also energetic) state of the dot, and processes affecting neither magnetization nor energy of the dot. The former (latter) processes will be referred to as inelastic (elastic) ones. The elastic cotunneling processes are fully coherent 27] and do not change the dot occupation probabilities. Contrarily, inelastic cotunneling influences the occupation numbers of the dot, and can take place only when the dot is occupied by a single electron - either spin-up or spin-down. Furthermore, one can also distinguish between single-barrier and double-barrier cotunneling. Only the latter processes contribute directly to the current. However, the inelastic single-barrier cotunneling processes can change the occupation probabilities, and consequently can also influence the electric current flowing through the system. In particular, inelastic single-barrier cotunneling processes which reverse spin of the dot play a significant role. This is because they can open system for more efficient tunneling processes, when the system is blocked by an electron of a given spin orientation residing on the dot 12 .

In the following we will consider two different situations. The first one corresponds to an empty dot (due to particle-hole symmetry the results can be adapted to the case of doubly occupied dot). The second situation is the case of a singly occupied dot.

\section{Cotunneling through an empty dot}

When the dot level is far above the Fermi energy of the leads, $\varepsilon_{\sigma} \gg k_{\mathrm{B}} T, \Gamma,|e V|$, there are no electrons on the quantum dot and electric current can flow only due to elastic cotunneling processes. Because of the particle-hole symmetry, a similar analysis can be directly performed for a doubly occupied dot, when $\varepsilon_{\sigma}+U \ll 0$ and $\left|\varepsilon_{\sigma}+U\right| \gg$ $k_{\mathrm{B}} T, \Gamma,|e V|$. As in the case of an empty dot, the current can then flow only due to elastic cotunneling.

\subsection{Theoretical description}

Electric current $I$ flowing from the left to right lead is given by

$$
I=-e \sum_{\sigma, \sigma^{\prime}=+,-}\left(\gamma_{\mathrm{LR}, 0}^{\sigma \Rightarrow \sigma^{\prime}}-\gamma_{\mathrm{RL}, 0}^{\sigma \Rightarrow \sigma^{\prime}}\right)
$$

with $\gamma_{\mathrm{LR}, \chi}^{\sigma \Rightarrow \sigma^{\prime}}$ being the elastic cotunneling rate for transition from the left to right leads, when the dot is in the state $\left|\Phi_{\chi}\right\rangle[\chi=0$ in Eq.(3)] and when a majority $(\sigma=+)$ or minority $(\sigma=-)$ electron of the left lead tunnels to majority $\left(\sigma^{\prime}=+\right)$ or minority $\left(\sigma^{\prime}=-\right)$ electron band in the right lead. Similarly, $\gamma_{\mathrm{RL}, 0}^{\sigma \Rightarrow \sigma^{\prime}}$ is the elastic cotunneling rate for transition from the right to left electrodes. In Eq. (3) $-e$ denotes the electron charge $(e>0)$.

The transition rate for electrons tunneling from the majority spin band in the left lead to the majority spin band in the right lead is given by the formula

$$
\begin{aligned}
& \gamma_{\mathrm{LR}, 0}^{+\Rightarrow+}=\frac{2 \pi}{\hbar} \iint d \varepsilon_{\mathrm{L}} d \varepsilon_{\mathrm{R}} \rho_{\mathrm{L}+} \rho_{\mathrm{R}+} \\
& \quad \times\left|T_{\mathrm{L}+}\right|^{2}\left|T_{\mathrm{R}+}\right|^{2} f\left(\varepsilon_{\mathrm{L}}\right)\left[1-f\left(\varepsilon_{\mathrm{R}}\right)\right] \\
& \quad \times\left[\frac{\cos \left(\varphi_{\mathrm{L}} / 2\right) \cos \left(\varphi_{\mathrm{R}} / 2\right)}{\varepsilon_{\mathrm{L}}+\mu_{\mathrm{L}}-\varepsilon_{\uparrow}}+\frac{\sin \left(\varphi_{\mathrm{L}} / 2\right) \sin \left(\varphi_{\mathrm{R}} / 2\right)}{\varepsilon_{\mathrm{L}}+\mu_{\mathrm{L}}-\varepsilon_{\downarrow}}\right]^{2} \\
& \quad \times \delta\left(\varepsilon_{\mathrm{L}}+\mu_{\mathrm{L}}-\varepsilon_{\mathrm{R}}-\mu_{\mathrm{R}}\right)
\end{aligned}
$$

with $\mu_{\mathrm{L}}\left(\mu_{\mathrm{R}}\right)$ denoting the electrochemical potential of the left (right) lead and $f(\varepsilon)$ being the Fermi-Dirac distribution function, $f(\varepsilon)=1 /\left[\exp \left(\varepsilon / k_{\mathrm{B}} T\right)+1\right]$. We assume $\mu_{\mathrm{L}}=-\mathrm{eV} / 2$ and $\mu_{\mathrm{R}}=e V / 2$, and the energy is measured from the Fermi level of the leads in equilibrium situation $(V=0)$. The integrals in Eq.(4) can be calculated quite easily using the contour integration method, as described in the Appendix. Following this procedure one finds

$$
\begin{aligned}
\gamma_{\mathrm{LR}, 0}^{+\Rightarrow+}= & \frac{\Gamma_{\mathrm{L}}^{+} \Gamma_{\mathrm{R}}^{+}}{h} f_{\mathrm{B}}\left(\mu_{\mathrm{R}}-\mu_{\mathrm{L}}\right)\left\{\cos ^{2} \frac{\varphi_{\mathrm{L}}}{2} \cos ^{2} \frac{\varphi_{\mathrm{R}}}{2} A_{2}\left(\varepsilon_{\uparrow}\right)\right. \\
& +\sin ^{2} \frac{\varphi_{\mathrm{L}}}{2} \sin ^{2} \frac{\varphi_{\mathrm{R}}}{2} A_{2}\left(\varepsilon_{\downarrow}\right) \\
& \left.+\frac{\sin \varphi_{\mathrm{L}} \sin \varphi_{\mathrm{R}}}{2\left(\varepsilon_{\uparrow}-\varepsilon_{\downarrow}\right)}\left[A_{1}\left(\varepsilon_{\uparrow}\right)-A_{1}\left(\varepsilon_{\downarrow}\right)\right]\right\}
\end{aligned}
$$

where $f_{\mathrm{B}}(\varepsilon)$ is the Bose function, $f_{\mathrm{B}}(\varepsilon)=1 /\left[\exp \left(\varepsilon / k_{\mathrm{B}} T\right)-\right.$ $1]$, and $A_{n}\left(\varepsilon_{\sigma}\right)=B_{n}\left(\varepsilon_{\sigma}-\mu_{\mathrm{R}}\right)-B_{n}\left(\varepsilon_{\sigma}-\mu_{\mathrm{L}}\right)$, with $B_{n}(x)$ defined as

$$
B_{n}(x)=\operatorname{Re} \frac{\mathrm{d}^{(n-1)}}{\mathrm{d}^{(n-1)} x} \Psi\left(\frac{1}{2}+\frac{\mathrm{i} x}{2 \pi k_{\mathrm{B}} T}\right) .
$$

Here $\Psi(z)$ is the digamma function. Similar formulae can also be derived for the other transition rates. 


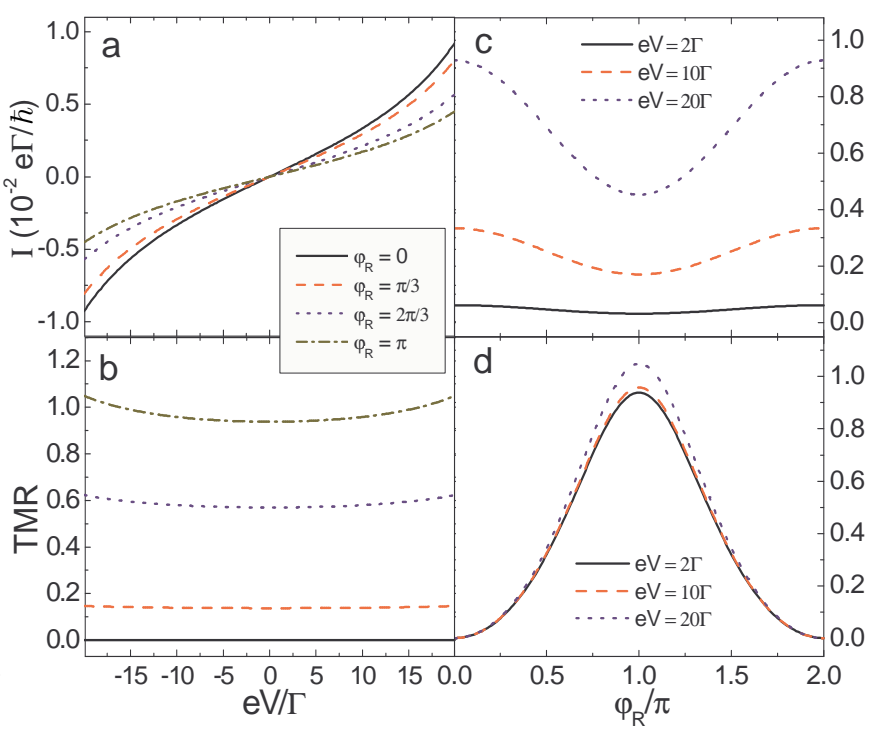

Fig. 2. (Color online) The cotunneling current (a,c) and the TMR effect (b,d) as a function of the bias voltage (left column) and the angle $\varphi_{\mathrm{R}}$ (right column). The parameters assumed for calculations are: $k_{\mathrm{B}} T=\Gamma, \varepsilon_{\uparrow}=18 \Gamma, \varepsilon_{\downarrow}=22 \Gamma, P_{\mathrm{L}}=P_{\mathrm{R}}=$ $0.5, \varphi_{\mathrm{L}}=0$.

Having found all the cotunneling rates, one can calculate the electric current,

$$
\begin{aligned}
I= & \frac{e \Gamma^{2}}{4 h}\left\{\left(1-P_{\mathrm{L}} \cos \varphi_{\mathrm{L}}\right)\left(1-P_{\mathrm{R}} \cos \varphi_{\mathrm{R}}\right) A_{2}\left(\varepsilon_{\downarrow}\right)\right. \\
& +\left(1+P_{\mathrm{L}} \cos \varphi_{\mathrm{L}}\right)\left(1+P_{\mathrm{R}} \cos \varphi_{\mathrm{R}}\right) A_{2}\left(\varepsilon_{\uparrow}\right) \\
& \left.+\frac{2 P_{\mathrm{L}} P_{\mathrm{R}} \sin \varphi_{\mathrm{L}} \sin \varphi_{\mathrm{R}}}{\varepsilon_{\downarrow}-\varepsilon_{\uparrow}}\left[A_{1}\left(\varepsilon_{\downarrow}\right)-A_{1}\left(\varepsilon_{\uparrow}\right)\right]\right\} .
\end{aligned}
$$

In the following we will present numerical results on the electric current and the associated magnetoresistance. The TMR effect is described quantitatively by the ratio

$$
\mathrm{TMR}=\frac{I_{\mathrm{P}}-I\left(\varphi_{\mathrm{L}}, \varphi_{\mathrm{R}}\right)}{I\left(\varphi_{\mathrm{L}}, \varphi_{\mathrm{R}}\right)},
$$

where $I\left(\varphi_{\mathrm{L}}, \varphi_{\mathrm{R}}\right)$ is the current flowing in the noncollinear configuration described by the angles $\varphi_{\mathrm{L}}$ and $\varphi_{\mathrm{R}}$, whereas $I_{\mathrm{P}}$ is the current flowing in the parallel configuration corresponding to $\varphi_{\mathrm{L}}=\varphi_{\mathrm{R}}=0$.

\subsection{Numerical results}

The formula (7) for electric current corresponds to the situation, where the magnetic moments of the leads and the effective molecular field acting on the dot are oriented arbitrarily in the plane of the structure. Further numerical analysis will be restricted, however, to the following two situations: (i) the magnetic moment of the left lead is parallel to the molecular field acting on the $\operatorname{dot}\left(\varphi_{\mathrm{L}}=0\right)$, while the magnetic moment of the right lead can have an arbitrary orientation, and (ii) the magnetic moments of both leads can rotate symmetrically in the opposite directions, $\varphi_{\mathrm{R}}=-\varphi_{\mathrm{L}}$. The case (i) corresponds to the situation when magnetic moment of one lead and magnetic moment of the layer producing the molecular field acting on the dot are fixed along the same direction. This can be achieved for instance by a common antiferromagnetic underlayer with strong exchange anisotropy at the antiferromagnet/ferromagnet interface. The situation (ii), in turn, corresponds to the case when both leads are equivalent and their magnetic moments can be rotated simultaneously by an external magnetic field. Now, we will analyze both situations in more detail and we begin with the case (i).

\subsubsection{Case (i): $\varphi_{\mathrm{L}}=0$}

When $\varphi_{\mathrm{L}}=0$, the electric current is given by the formula

$$
\begin{aligned}
I= & \frac{e \Gamma^{2}}{4 h}\left[\left(1-P_{\mathrm{L}}\right)\left(1-P_{\mathrm{R}} \cos \varphi_{\mathrm{R}}\right) A_{2}\left(\varepsilon_{\downarrow}\right)\right. \\
& \left.+\left(1+P_{\mathrm{L}}\right)\left(1+P_{\mathrm{R}} \cos \varphi_{\mathrm{R}}\right) A_{2}\left(\varepsilon_{\uparrow}\right)\right],
\end{aligned}
$$

which follows directly from Eq. (7). In Figure 2 we show the current $(\mathrm{a}, \mathrm{c})$ flowing through the system and the corresponding TMR $(\mathrm{b}, \mathrm{d})$ as a function of the bias voltage for several values of angle $\varphi_{R}$, and as a function of the angle $\varphi_{\mathrm{R}}$ for several values of the bias voltage. The current decreases and TMR increases as the angle $\varphi_{\mathrm{R}}$ varies from $\varphi_{\mathrm{R}}=0$ to $\varphi_{\mathrm{R}}=\pi$, which corresponds to the transition from parallel to antiparallel magnetic configurations. Parts (c) and (d) show explicitly this angular dependence. Both the current and TMR vary monotonously with $\varphi_{\mathrm{R}}$ (for $0 \leq \varphi_{\mathrm{R}} \leq \pi$ ) and electric current reaches minimum, while TMR maximum, at $\varphi_{\mathrm{R}}=\pi$, i.e., in the antiparallel configuration. Such a behavior is typical of normal spin valves and results from spin asymmetry in tunneling processes. It is also worth noting that TMR is only weakly dependent on the bias voltage [see Fig. 2(b)].

Assuming the same spin polarization of the leads, $P_{\mathrm{L}}=$ $P_{\mathrm{R}}=P$, one finds the following explicit formula for TMR in the zero bias and zero temperature limits:

$\operatorname{TMR}=\frac{\left(1-\cos \varphi_{R}\right) P\left[(1+P) \varepsilon_{\downarrow}^{2}-(1-P) \varepsilon_{\uparrow}^{2}\right]}{(1+P)\left(1+P \cos \varphi_{R}\right) \varepsilon_{\downarrow}^{2}+(1-P)\left(1-P \cos \varphi_{R}\right) \varepsilon_{\uparrow}^{2}}$

The above formula describes the angular variation of TMR and shows explicitly that TMR reaches maximum for $\varphi=$ $\pi$. This maximum value is given by the expression

$$
\mathrm{TMR}^{(\max )}=\frac{2 P}{1-P^{2}}\left[P+\frac{\varepsilon_{\downarrow}^{2}-\varepsilon_{\uparrow}^{2}}{\varepsilon_{\uparrow}^{2}+\varepsilon_{\downarrow}^{2}}\right] .
$$

The first term in the bracket of the above equation gives the Julliere's value of TMR, whereas the second term describes enhancement of the tunnel magnetoresistance due to level splitting. Such an enhancement of TMR may be of some interest from the application point of view. 


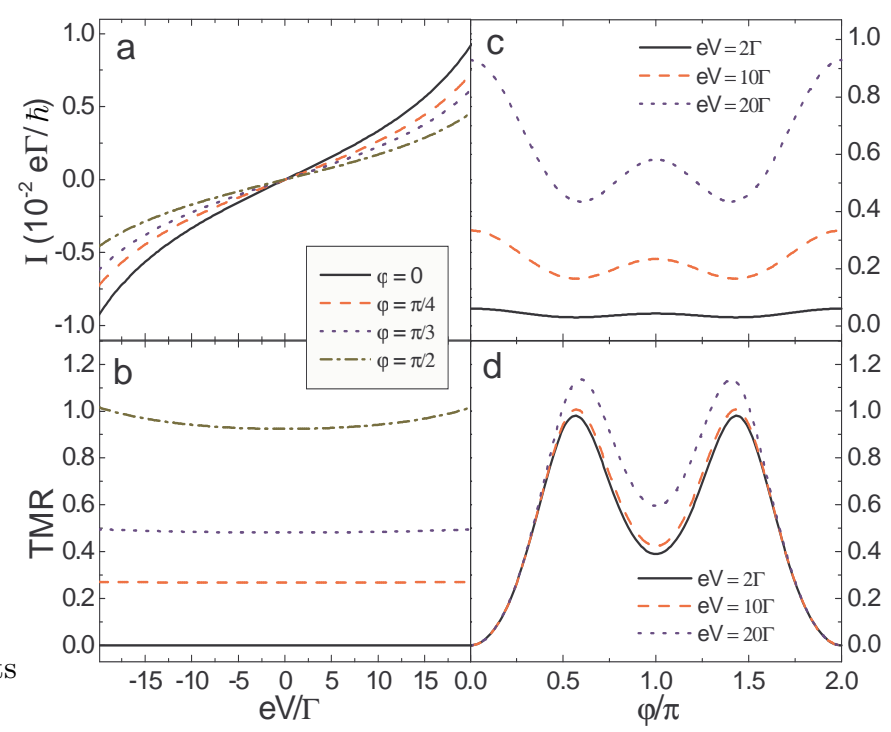

Fig. 3. (Color online) The cotunneling current $(\mathrm{a}, \mathrm{c})$ and the TMR effect (b,d) as a function of the bias voltage (left column) and as a function of $\varphi=\varphi_{\mathrm{R}}=-\varphi_{\mathrm{L}}$ (right column). The other parameters are the same as in Fig. 2.

\subsubsection{Case (ii): $\varphi_{\mathrm{R}}=-\varphi_{\mathrm{L}}$}

Consider now the situation (ii), when $\varphi_{\mathrm{R}}=-\varphi_{\mathrm{L}} \equiv \varphi$. The magnetic moment of the left lead rotates now together with the magnetic moment of the right lead, but in the opposite direction. The electric current is then given by Eq. (7) with $\varphi_{\mathrm{R}}=\varphi$ and $\varphi_{\mathrm{L}}=-\varphi$. The corresponding bias and angular dependence of the cotunneling current and TMR is displayed in Fig. 3]

It is interesting to note that the angular dependence of electric current and TMR differs now from that found above for the situation (i). In order to understand this difference one should take into account the fact that $\varphi_{\mathrm{R}}=$ $\varphi_{\mathrm{L}}=0$ corresponds to the parallel configuration, whereas the situation with $\varphi_{\mathrm{R}}=-\varphi_{\mathrm{L}}=\pi / 2$ corresponds to the antiparallel configuration with magnetic moments oriented perpendicularly to the molecular field acting on the dot. It is also worth noting that electric current reaches minimum and TMR maximum not exactly at $\varphi_{\mathrm{R}}=-\varphi_{\mathrm{L}}=\pi / 2$, but for the configuration which is close to the antiparallel one. Moreover, position of these minima and maxima depends on the bias voltage, as shown in Fig. 3(c,d). In turn, the case $\varphi_{\mathrm{R}}=-\varphi_{\mathrm{L}}=\pi$ corresponds again to the parallel configuration, but with the magnetic moments of the electrodes being antiparallel to the molecular field. This leads to a local maximum of electric current (minimum in $\mathrm{TMR}$ ) at $\varphi_{\mathrm{R}}=-\varphi_{\mathrm{L}}=\pi$. It is interesting to note that the two parallel configurations (aligned and anti-aligned with respect to the molecular field) are not equivalent, and consequently the corresponding currents and also TMR values are not equal.

When assuming equal spin polarizations of the leads and low bias and temperature limits, one finds that the TMR effect at $\varphi_{\mathrm{R}}=-\varphi_{\mathrm{L}}=\pi$ (which corresponds to its local minimum) is given by

$$
\operatorname{TMR}^{(\min )}=\frac{4 P\left(\varepsilon_{\downarrow}^{2}-\varepsilon_{\uparrow}^{2}\right)}{(1+P)^{2} \varepsilon_{\uparrow}^{2}+(1-P)^{2} \varepsilon_{\downarrow}^{2}} .
$$

In the same limit and for a nonzero spin polarization $P$, one can show that the two maxima of TMR appear at $\varphi=\arccos \alpha$ and $\varphi=2 \pi-\arccos \alpha$, with $\alpha=-\Delta / P\left(\varepsilon_{\uparrow}+\right.$ $\left.\varepsilon_{\downarrow}\right)$. In the limit of $P=0$ TMR vanishes by definition. In the case when $|\Delta|<P\left(\varepsilon_{\uparrow}+\varepsilon_{\downarrow}\right)$, the maxima appear approximately at $\varphi_{\mathrm{R}}=-\varphi_{\mathrm{L}}=\pi / 2$ and $\varphi_{\mathrm{R}}=-\varphi_{\mathrm{L}}=$ $3 \pi / 2$. The corresponding TMR value is then equal

$$
\mathrm{TMR}^{(\max )}=\frac{P\left[(2+P) \varepsilon_{\downarrow}-(2-P) \varepsilon_{\uparrow}\right]\left(\varepsilon_{\uparrow}+\varepsilon_{\downarrow}\right)}{\varepsilon_{\uparrow}^{2}+\varepsilon_{\downarrow}^{2}-2 P^{2} \varepsilon_{\uparrow} \varepsilon_{\downarrow}} .
$$

\section{Cotunneling through a singly occupied dot}

By applying an external gate voltage to the dot, one can tune position of the level energy and this way also the dot occupation. When $\varepsilon_{\sigma}$ is negative and $\varepsilon_{\sigma}+U$ positive, the dot is singly occupied at equilibrium for $\Gamma, k_{\mathrm{B}} T \ll$ $\left|\varepsilon_{\sigma}\right|,\left|\varepsilon_{\sigma}+U\right|$, and the system is in the Coulomb blockade regime. As before, we will consider the two situations (i) and (ii) defined in the previous section.

\subsection{Theoretical description}

In the case studied in the preceding section the dot was empty, and the second-order current was mediated only by elastic cotunneling processes. When the dot is singly occupied, the cotunneling current can also flow due to inelastic cotunneling, in which the electrons tunneling to and off the dot have opposite spin orientations (and consequently also different energies due to the level spin-splitting). These inelastic cotunneling processes determine the occupation numbers of the dot.

The probabilities $p_{\uparrow}$ and $p_{\downarrow}$, that the dot is occupied either by a spin-up or spin-down electron, can be calculated from the following stationary master equation:

$$
0=\sum_{\alpha, \beta=\mathrm{L}, \mathrm{R}}\left(-\gamma_{\alpha \beta, \uparrow \Rightarrow \downarrow} p_{\uparrow}+\gamma_{\alpha \beta, \downarrow \Rightarrow \uparrow} p_{\downarrow}\right),
$$

and taking into account also the normalization condition, $p_{\uparrow}+p_{\downarrow}=1$. Here, $\gamma_{\alpha \beta, \sigma \Rightarrow \bar{\sigma}}$ denotes the rate of inelastic cotunneling from lead $\alpha$ to lead $\beta$ with the simultaneous change of the dot spin from $\sigma$ to $\bar{\sigma} \equiv-\sigma$. The inelastic cotunneling processes can take place through one of the two virtual states, $\left|\Phi_{0}\right\rangle$ and $\left|\Phi_{\mathrm{d}}\right\rangle$, which correspond to the empty and doubly occupied dot, respectively. The corresponding energies are $\varepsilon_{0}=0$ for the empty state and $\varepsilon_{\mathrm{d}}=\varepsilon_{\uparrow}+\varepsilon_{\downarrow}+U$ for the doubly occupied state.

The rate of inelastic processes which transfer a spinmajority electron from the left lead to the spin-down level 
of the dot and a spin-up electron from the dot to the spinmajority electron band in the right lead is given by

$$
\begin{aligned}
\gamma_{\mathrm{L}, \uparrow \Rightarrow \downarrow}^{+\Rightarrow+}= & \frac{2 \pi}{\hbar} \iint d \varepsilon_{\mathrm{L}} d \varepsilon_{\mathrm{R}} \rho_{\mathrm{L}+} \rho_{\mathrm{R}+}\left|T_{\mathrm{L}+}\right|^{2}\left|T_{\mathrm{R}+}\right|^{2} \\
& \times \sin ^{2} \frac{\varphi_{\mathrm{L}}}{2} \cos ^{2} \frac{\varphi_{\mathrm{R}}}{2} f\left(\varepsilon_{\mathrm{L}}\right)\left[1-f\left(\varepsilon_{\mathrm{R}}\right)\right] \\
& \times\left(\frac{1}{\varepsilon_{\mathrm{L}}+\mu_{\mathrm{L}}-\varepsilon_{\downarrow}-U}-\frac{1}{\varepsilon_{\mathrm{R}}+\mu_{\mathrm{R}}-\varepsilon_{\uparrow}}\right)^{2} \\
& \times \delta\left(\varepsilon_{\mathrm{L}}+\mu_{\mathrm{L}}-\varepsilon_{\mathrm{R}}-\mu_{\mathrm{R}}+\varepsilon_{\uparrow}-\varepsilon_{\downarrow}\right) .
\end{aligned}
$$

The total rate of inelastic cotunneling from the left to right leads, which changes the dot state from $\left|\Phi_{\uparrow}\right\rangle$ to $\left|\Phi_{\downarrow}\right\rangle$, can be found by summing up over the spin-majority and spin-minority electrons,

$$
\gamma_{\mathrm{LR}, \uparrow \Rightarrow \downarrow}=\sum_{\sigma, \sigma^{\prime}=+,-} \gamma_{\mathrm{LR}, \uparrow \Rightarrow \downarrow}^{\sigma \Rightarrow \sigma^{\prime}}
$$

The corresponding analytical expression can be derived in a similar way as in the case of elastic cotunneling described in the previous section and takes the form

$$
\begin{aligned}
& \gamma_{\mathrm{LR}, \uparrow \Rightarrow \downarrow}=\frac{f_{\mathrm{B}}\left(\mu_{\mathrm{R}}-\mu_{\mathrm{L}}-\varepsilon_{\uparrow}+\varepsilon_{\downarrow}\right)}{h} \\
& \quad \times\left(\Gamma_{\mathrm{L}}^{+} \Gamma_{\mathrm{R}}^{+} \sin ^{2} \frac{\varphi_{\mathrm{L}}}{2} \cos ^{2} \frac{\varphi_{\mathrm{R}}}{2}+\Gamma_{\mathrm{L}}^{+} \Gamma_{\mathrm{R}}^{-} \sin ^{2} \frac{\varphi_{\mathrm{L}}}{2} \sin ^{2} \frac{\varphi_{\mathrm{R}}}{2}\right. \\
& \left.+\Gamma_{\mathrm{L}}^{-} \Gamma_{\mathrm{R}}^{+} \cos ^{2} \frac{\varphi_{\mathrm{L}}}{2} \cos ^{2} \frac{\varphi_{\mathrm{R}}}{2}+\Gamma_{\mathrm{L}}^{-} \Gamma_{\mathrm{R}}^{-} \cos ^{2} \frac{\varphi_{\mathrm{L}}}{2} \sin ^{2} \frac{\varphi_{\mathrm{R}}}{2}\right) \\
& \quad \times\left\{B_{2}\left(\varepsilon_{\uparrow}-\mu_{\mathrm{R}}\right)-B_{2}\left(\varepsilon_{\downarrow}-\mu_{\mathrm{L}}\right)\right. \\
& +B_{2}\left(\varepsilon_{\uparrow}+U-\mu_{\mathrm{R}}\right)-B_{2}\left(\varepsilon_{\downarrow}+U-\mu_{\mathrm{L}}\right) \\
& +\frac{2}{U}\left[B_{1}\left(\varepsilon_{\uparrow}-\mu_{\mathrm{R}}\right)-B_{1}\left(\varepsilon_{\downarrow}-\mu_{\mathrm{L}}\right)\right. \\
& \left.\left.-B_{1}\left(\varepsilon_{\uparrow}+U-\mu_{\mathrm{R}}\right)+B_{1}\left(\varepsilon_{\downarrow}+U-\mu_{\mathrm{L}}\right)\right]\right\} .
\end{aligned}
$$

The cotunneling rate $\gamma_{\mathrm{LR}, \downarrow \Rightarrow \uparrow}$ can be calculated in a similar way. In turn, the rate of elastic cotunneling can be found as described in section III.

Having calculated the cotunneling rates and the occupation probabilities, one can determine the current flowing from the left to right leads. The contributions $I_{\text {inel }}$ and $I_{\mathrm{el}}$ due to inelastic and elastic cotunneling, respectively, are given by

$$
\begin{aligned}
I_{\text {inel }} & =-e \sum_{\sigma=\uparrow, \downarrow}\left(\gamma_{\mathrm{LR}, \sigma \Rightarrow \bar{\sigma}}-\gamma_{\mathrm{RL}, \sigma \Rightarrow \bar{\sigma}}\right) p_{\sigma}, \\
I_{\mathrm{el}} & =-e \sum_{\sigma=\uparrow, \downarrow} \sum_{\sigma^{\prime}, \sigma^{\prime \prime}=+,-}\left(\gamma_{\mathrm{LR}, \sigma}^{\sigma^{\prime} \Rightarrow \sigma^{\prime \prime}}-\gamma_{\mathrm{RL}, \sigma}^{\sigma^{\prime} \Rightarrow \sigma^{\prime \prime}}\right) p_{\sigma} .
\end{aligned}
$$

The total cotunneling current $I$ is then equal

$$
I=I_{\text {inel }}+I_{\mathrm{el}} .
$$

The analytical expression for the current in the case of a singly occupied dot is cumbersome and will not be presented here.

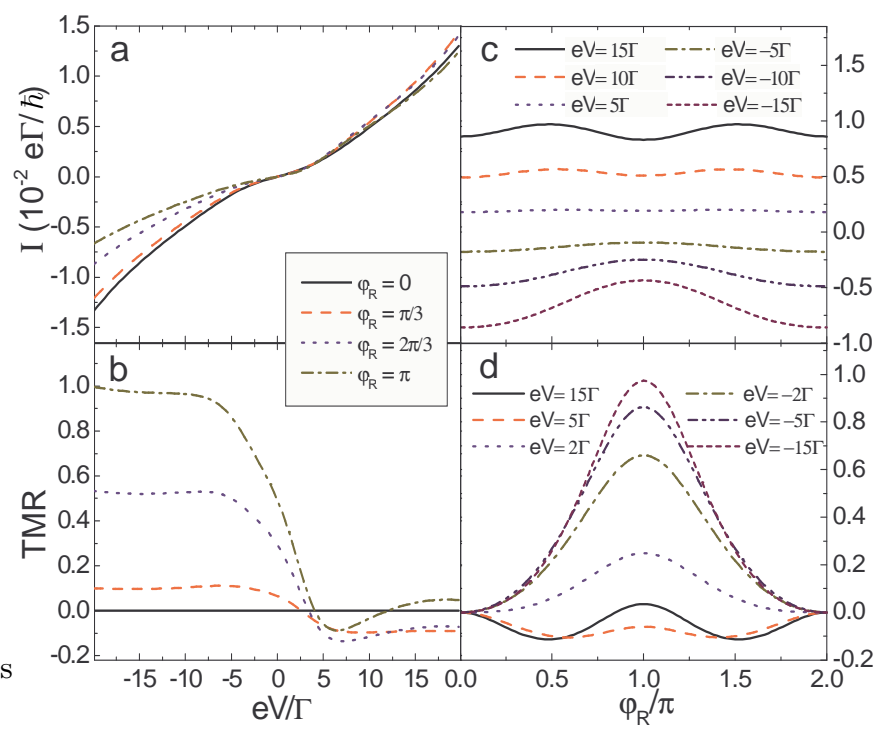

Fig. 4. (Color online) The cotunneling current (a,c) and the TMR effect (b,d) as a function of the bias voltage (left column) and the angle $\varphi_{\mathrm{R}}$ (the right column). The parameters assumed for calculations are: $k_{\mathrm{B}} T=\Gamma, \varepsilon_{\uparrow}=-22 \Gamma, \varepsilon_{\downarrow}=-18 \Gamma, U=$ $40 \Gamma, P_{\mathrm{L}}=P_{\mathrm{R}}=0.5, \varphi_{\mathrm{L}}=0$.

\subsection{Numerical results}

As far as physics is concerned, the situation with singly occupied dot becomes more interesting. As before, we will analyze the two situations (i) and (ii).

\subsubsection{Case (i): $\varphi_{\mathrm{L}}=0$}

When the magnetic moment of the left electrode is fixed $\left(\varphi_{\mathrm{L}}=0\right)$ and the magnetic moment of the right lead is free to rotate, the angular and bias dependence of electric current and TMR is shown in Fig. 4. Except for the parallel configuration, the current-voltage curves are now asymmetric with respect to the bias reversal [see Fig. 4(a)]. This asymmetry also leads to related asymmetric behavior of TMR [Fig. 4(b)]. Moreover, for positive bias voltage, the TMR effect can change sign and become negative in a certain range of the bias and angle values. Such an asymmetry in transport characteristics with respect to the bias reversal is of some importance for applications, particularly when the current is significantly suppressed for one bias polarization (diode behavior).

In order to account for the bias asymmetry, let us consider only the antiparallel configuration. One should then realize that owing to the level splitting, the single-barrier inelastic cotunneling processes can occur only when the dot is occupied by a spin-down electron. This follows simply from the energy conservation rule. Thus, the singlebarrier processes can assist the fastest double-barrier cotunneling processes (spin-up electron tunnels through the left barrier and spin-down electron tunnels through the right barrier), but only for positive bias. This is because the fastest processes can occur when the dot is occupied 


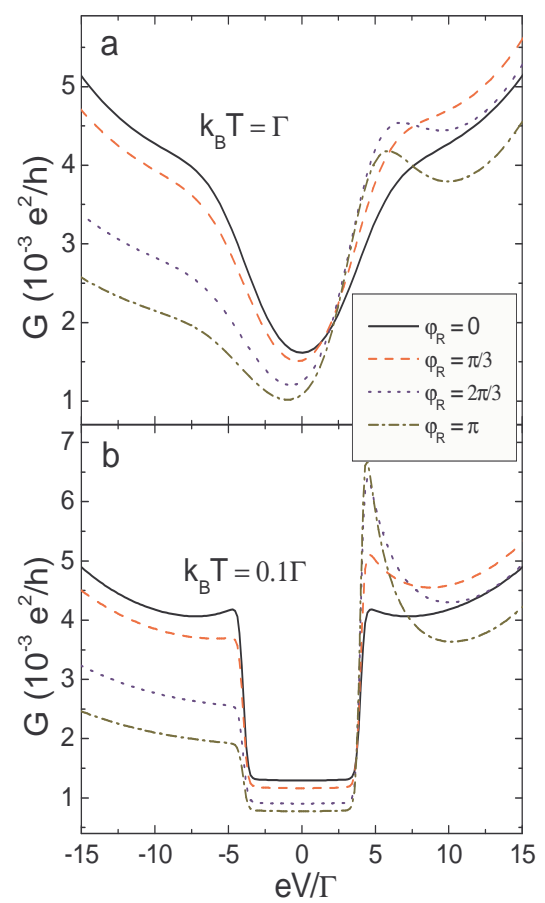

Fig. 5. (Color online) Differential conductance corresponding to the situation shown in Fig. 4(a), calculated for two different temperatures.

by a spin-down electron for negative bias and by a spin-up electron for positive bias. From this follows that the conductance is larger for positive than for negative bias voltage. This is indeed the case in the characteristics shown in Fig. 4(a). The above described mechanism of the asymmetry with respect to the bias reversal does not hold when magnetic moments of the two leads are parallel, so the corresponding current-voltage curve is symmetrical.

To demonstrate the above described asymmetry more clearly, the corresponding differential conductance is displayed in Fig. 5(a). The asymmetry for $\varphi_{\mathrm{R}}>0$ is clearly evident. Besides the asymmetry, an additional interesting feature of the differential conductance is also visible, namely the characteristic deep in the small bias regime. This deep is a consequence of the suppression of inelastic double-barrier cotunneling events when $|e V|<|\Delta|$. For $|e V|>|\Delta|$, the inelastic cotunneling processes are allowed, leading to an enhanced conductance. The asymmetry and zero bias anomaly are even more evident at lower temperature, as shown in Fig. 5(b). Such a suppression of the inelastic cotunneling events at small bias was used recently as a spectroscopic tool to determine spin splitting of the dot level and the corresponding $g$-factor [28.

The angular variation of electric current and TMR reveals further new features. For negative bias there is a maximum of absolute value of electric current in the parallel configuration and a minimum in the antiparallel configuration. For positive bias, however, the electric current has a maximum for noncollinear configuration, as depicted in Fig. 4(c). The nonmonotonous variation of electric current with the angle $\varphi_{\mathrm{R}}$ leads to the corresponding

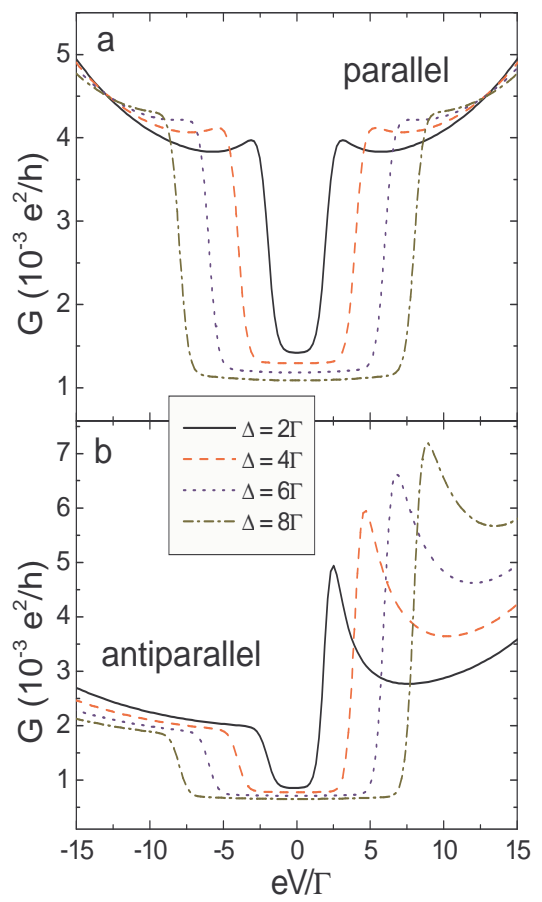

Fig. 6. (Color online) Differential conductance for different values of the level splitting for the parallel (a) and antiparallel (b) magnetic configurations. The parameters are: $k_{\mathrm{B}} T=0.2 \Gamma$, $\varepsilon_{\uparrow}=\varepsilon-\Delta / 2, \varepsilon_{\downarrow}=\varepsilon+\Delta / 2, \varepsilon=-20 \Gamma, U=40 \Gamma$, and $P_{\mathrm{L}}=P_{\mathrm{R}}=0.5$.

nonmonotonous variation of TMR, shown in Fig. 4(d). Furthermore, TMR may now become negative, as already mentioned before.

Numerical results presented so far were shown for a single value of the level splitting, $\Delta=4 \Gamma$. From the experimental point of view, variation of the conductance as a function of the level splitting (induced for instance by a strong external magnetic field), allows one to determine some interesting transport and spectroscopic characteristics. Therefore, in Fig. 6 we show the differential conductance for different splitting of the dot level in the parallel and antiparallel configurations. By measuring width of the conductance deep, one can determine for instance the spectroscopic $g$-factor [28].

In the case of a deep Coulomb blockade regime and $|e V|, k_{\mathrm{B}} T \ll|\Delta|$, one can derive an approximate formula for the deep in differential conductance due to the suppression of inelastic cotunneling. Writing $\varepsilon_{\uparrow}=\varepsilon-\Delta / 2$ and $\varepsilon_{\downarrow}=\varepsilon+\Delta / 2$ one finds then the following expression:

$$
\begin{aligned}
G= & \frac{\Gamma^{2} e^{2}}{4 h}\left[\frac{\left(1+P_{\mathrm{L}} \cos \varphi_{\mathrm{L}}\right)\left(1+P_{\mathrm{R}} \cos \varphi_{\mathrm{R}}\right)}{(\varepsilon-\Delta / 2)^{2}}\right. \\
& +\frac{\left(1-P_{\mathrm{L}} \cos \varphi_{\mathrm{L}}\right)\left(1-P_{\mathrm{R}} \cos \varphi_{\mathrm{R}}\right)}{(\varepsilon+U+\Delta / 2)^{2}} \\
& \left.-\frac{8 P_{\mathrm{L}} P_{\mathrm{R}} \sin \varphi_{\mathrm{L}} \sin \varphi_{\mathrm{R}}}{(\varepsilon-\Delta / 2)(\varepsilon+U+\Delta / 2)}\right],
\end{aligned}
$$

which is valid for arbitrary magnetic configurations. This expression approximates the plateaus shown in Fig. 5. 
When assuming $\varphi_{\mathrm{L}}=\varphi_{\mathrm{R}}=0$ (which corresponds to the parallel configuration), Eq. (21) simplifies to the following form

$$
G_{\mathrm{P}}=\frac{\Gamma^{2} e^{2}}{4 h}\left[\frac{\left(1+P_{\mathrm{L}}\right)\left(1+P_{\mathrm{R}}\right)}{(\varepsilon-\Delta / 2)^{2}}+\frac{\left(1-P_{\mathrm{L}}\right)\left(1-P_{\mathrm{R}}\right)}{(\varepsilon+U+\Delta / 2)^{2}}\right],
$$

whereas for the antiparallel alignment $\left(\varphi_{\mathrm{L}}=0, \varphi_{\mathrm{R}}=\pi\right)$ it becomes

$$
G_{\mathrm{AP}}=\frac{\Gamma^{2} e^{2}}{4 h}\left[\frac{\left(1+P_{\mathrm{L}}\right)\left(1-P_{\mathrm{R}}\right)}{(\varepsilon-\Delta / 2)^{2}}+\frac{\left(1-P_{\mathrm{L}}\right)\left(1+P_{\mathrm{R}}\right)}{(\varepsilon+U+\Delta / 2)^{2}}\right] .
$$

The above two expressions describe the plateaus in differential conductance shown in Fig. 6. It is also worth noting that generally $G_{\mathrm{P}}>G_{\mathrm{AP}}$. Furthermore, both $G_{\mathrm{P}}$ and $G_{\mathrm{AP}}$ vary monotonously with spin polarization of the leads - in the case of nonmagnetic leads $\left(P_{\mathrm{L}}=P_{\mathrm{R}}=0\right)$ $G_{\mathrm{P}}=G_{\mathrm{AP}}$, whereas for $P_{\mathrm{L}}=P_{\mathrm{R}}=1$ (which corresponds to half-metallic leads) $G_{\mathrm{P}}$ is maximal and $G_{\mathrm{AP}}=0$.

\subsubsection{Case (ii): $\varphi_{\mathrm{R}}=-\varphi_{\mathrm{L}}$}

Transport characteristics in the second situation, i.e., for the case when $\varphi_{\mathrm{R}}=-\varphi_{\mathrm{L}}=\varphi$, are displayed in Fig. 7. One can note that the current is now almost independent of the magnetic configuration. Nevertheless, the angular dependence of the current becomes more visible in the corresponding differential conductance, plotted in Fig. 8(a) for different values of the angle $\varphi$, and also in Fig. 8(b) for the same situation, but for much lower temperature. The cotunneling gap due to suppression of the inelastic processes is also clearly visible. The deep in differential conductance for $|e V| \ll k_{\mathrm{B}} T,|\Delta|$ is given by Eq. (21). Since the system

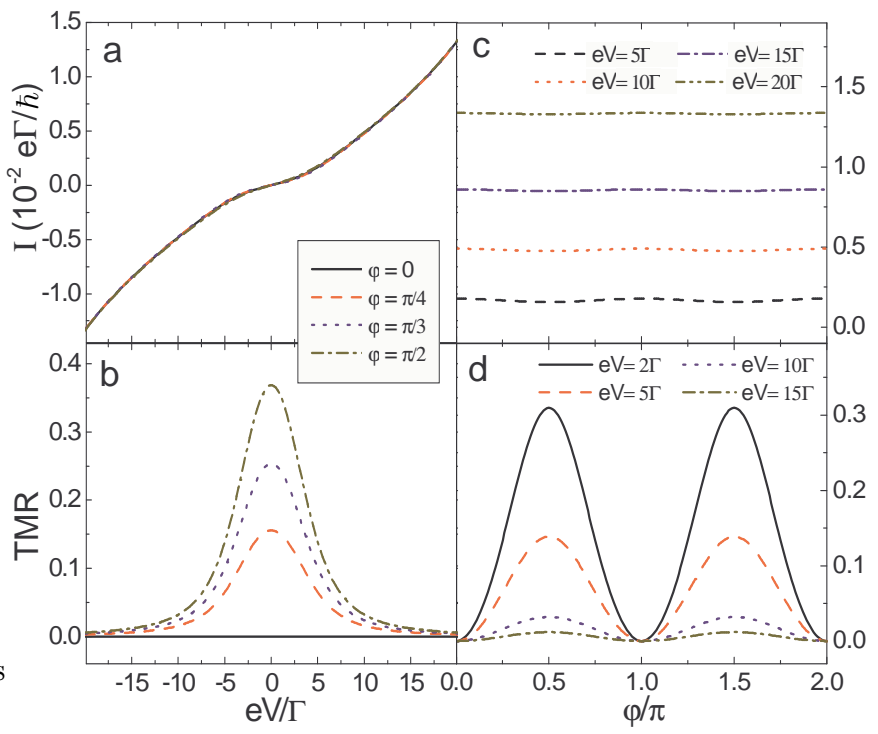

Fig. 7. (Color online) The cotunneling current (a,c) and the TMR effect (b,d) as a function of the bias voltage (left column) and $\varphi=\varphi_{\mathrm{R}}=-\varphi_{\mathrm{L}}$ (right column). The other parameters are the same as in Fig. 4.

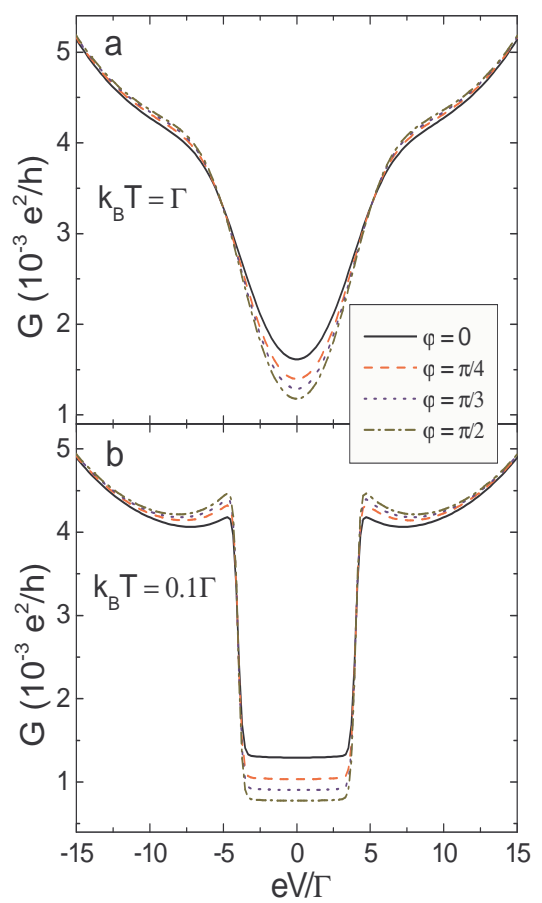

Fig. 8. (Color online) Differential conductance for the situation shown in Fig. 7(a), calculated for two indicated temperatures.

is now symmetric, the current-voltage curves (and consequently also the differential conductance) are symmetric with respect to the bias reversal.

The TMR effect reaches maximum in the zero bias limit, $V=0$, as shown in Fig. 7(b). In turn, angular variation of TMR reveals two maxima (and also two minima), similarly as it was in the case of empty dot, but now the maxima appear strictly for $\varphi=\pi / 2$ and $\varphi=3 \pi / 2$. On the other hand, one minimum of TMR occurs at $\varphi_{\mathrm{R}}=\varphi_{\mathrm{L}}=0$, where TMR vanishes by definition. Tunnel magnetoresistance vanishes also in the second parallel configuration, when both magnetizations are antiparallel to the molecular field $\left(\varphi_{\mathrm{R}}=-\varphi_{\mathrm{L}}=\pi\right)$. This is due to the fact that the parameters assumed for numerical calculations correspond to a symmetrical Anderson model, i.e., $U=-\varepsilon_{\uparrow}-\varepsilon_{\downarrow}$. When the system becomes asymmetric, e.g., when $U$ increases (decreases) while the other parameters are constant, the minimum in TMR at $\varphi=\pi$ is shifted down (up) and when $U \gg-\varepsilon_{\uparrow}-\varepsilon_{\downarrow}$, TMR has only one maximum at $\varphi=\pi$, as shown in Fig. 9(b). On the other hand, if $U<-\varepsilon_{\uparrow}-\varepsilon_{\downarrow}$, the minimum in tunnel magnetoresistance at $\varphi=\pi$ becomes negative, which is shown in Fig. 9(b) for $U=35 \Gamma$. Thus, by changing the model parameters one may significantly enhance or reduce the TMR effect.

In the limit of $|e V|, k_{\mathrm{B}} T \ll|\Delta|$ and for $P_{\mathrm{L}}=P_{\mathrm{R}}=P$, the TMR ratio for $\varphi_{\mathrm{R}}=-\varphi_{\mathrm{L}}=\pi$ can be expressed as

$$
\mathrm{TMR}=\frac{4 P(2 \varepsilon+U)(U+\Delta)}{(1-P)^{2}(\varepsilon+U+\Delta / 2)^{2}+(1+P)^{2}(\varepsilon-\Delta / 2)^{2}} .
$$




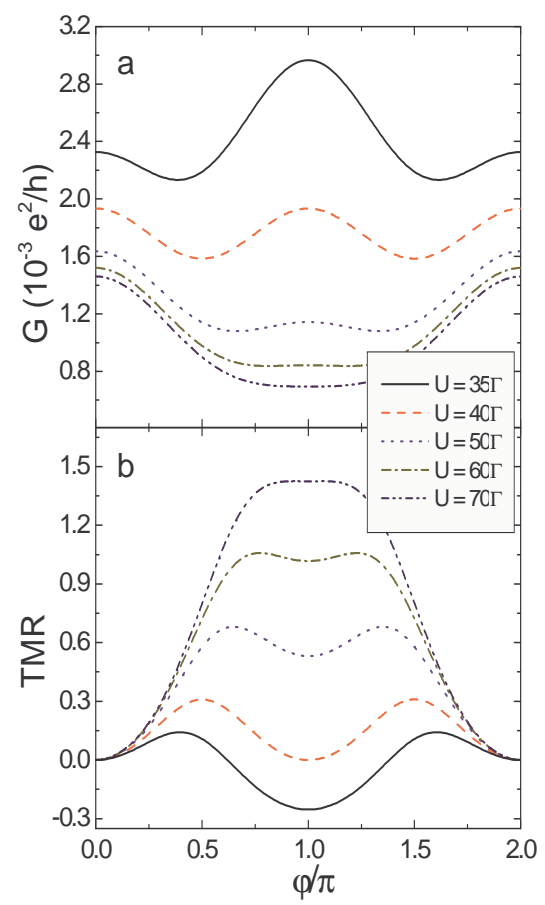

Fig. 9. (Color online) The angular dependence of the differential conductance and TMR for several values of the Coulomb interaction parameter $U$ at the bias voltage $e V=2 \Gamma$. The other parameters are the same as in Fig. 7.

From the above expression follows that the sign of TMR at $\varphi=\pi$ depends on the ratio $\varepsilon / U$. If $U=-2 \varepsilon$, TMR vanishes, whereas for $U \gtrless-2 \varepsilon$, TMR is positive (negative). In the case of $U \gg-2 \varepsilon$, the TMR effect is given by $4 P /(1-P)^{2}$.

The corresponding behavior of the differential conductance is shown in Fig.9(a). Now, the maximum in the conductance at $\varphi=\pi$ for a symmetric model changes into a minimum for $U \gg-\varepsilon_{\uparrow}-\varepsilon_{\downarrow}$.

\section{Conclusions}

We have considered analytically and numerically cotunneling current and associated tunnel magnetoresistance through a single-level quantum dot coupled to two external ferromagnetic leads. The dot level was assumed to be spin-split due to an effective molecular field originating from a magnetic substrate on which the dot is deposited, and the splitting was assumed to be larger than the characteristic parameter $\Gamma$ (level width) describing the dot-lead interaction. Although the general formulae were derived for arbitrary in-plane orientation of the molecular field and of the magnetic moments of the leads, detailed numerical analysis was performed for two particular configurations, which seem to be of particular interest. At this point we would like to note, that a related problem has been recently studied by Pedersen et al. 21, who considered cotunneling current in a similar system, but for different magnetic geometry. In our case all magnetic mo- ments and molecular field were in plane of the structure, whereas in their report magnetic moments were parallel while magnetic field was tilted out of the plane.

We have found several interesting features in the angular and bias dependence of transport characteristics. In the case of an empty dot, TMR was found to be roughly independent of the bias voltage, but strongly dependent on the angle between magnetic moments. When magnetic moments of both leads rotate in opposite directions, both electric current and TMR vary nonmonotonously with increasing angle between the magnetic moments of the leads, and maximum of TMR may occur at a noncollinear configuration. For a singly occupied dot and for the case (i) $\left(\varphi_{\mathrm{L}}=0\right)$, we found strong asymmetry in electric current and TMR with respect to the bias reversal, which disappears for the case (ii) $\left(\varphi_{\mathrm{R}}=-\varphi_{\mathrm{L}}\right)$. This diode-like behavior of the current-voltage characteristics may be of some interest from the application point of view. Moreover, the asymmetry in current-voltage curves leads to associated asymmetry in TMR which may become even negative for one bias polarization. An important and interesting result is also an enhancement of TMR due to the dot level splitting. Finally, we have also demonstrated numerically and analytically the evolution of the cotunneling gap with the splitting of the dot level and magnetic configuration of the system.

The work was supported by the Polish State Committee for Scientific Research through the projects PBZ/KBN/044/P03/200 and 2 P03B 116 25. The authors acknowledge discussions with Jürgen König and Jan Martinek.

\section{A Calculation of cotunneling rate}

Here we present some details of the calculation of cotunneling rates. As an example we consider the rate given by Eq. (4). Using the properties of the delta-Dirac function it can be written as

$$
\begin{aligned}
\gamma_{\mathrm{LR}, 0}^{+\Rightarrow+}= & \frac{\Gamma_{\mathrm{L}}^{+} \Gamma_{\mathrm{R}}^{+}}{h} \int d \varepsilon f(\varepsilon)\left[1-f\left(\varepsilon+\mu_{\mathrm{L}}-\mu_{\mathrm{R}}\right)\right] \\
& \times\left[\frac{\cos ^{2}\left(\varphi_{\mathrm{L}} / 2\right) \cos ^{2}\left(\varphi_{\mathrm{R}} / 2\right)}{\left(\varepsilon+\mu_{\mathrm{L}}-\varepsilon_{\uparrow}\right)^{2}}\right. \\
& +\frac{\sin \varphi_{\mathrm{L}} \sin \varphi_{\mathrm{R}}}{2\left(\varepsilon_{\uparrow}-\varepsilon_{\downarrow}\right)}\left(\frac{1}{\varepsilon+\mu_{\mathrm{L}}-\varepsilon_{\uparrow}}-\frac{1}{\varepsilon+\mu_{\mathrm{L}}-\varepsilon_{\downarrow}}\right) \\
& \left.+\frac{\sin ^{2}\left(\varphi_{\mathrm{L}} / 2\right) \sin ^{2}\left(\varphi_{\mathrm{R}} / 2\right)}{\left(\varepsilon+\mu_{\mathrm{L}}-\varepsilon_{\downarrow}\right)^{2}}\right] .
\end{aligned}
$$

Then, taking into account the identity

$$
f(\varepsilon)\left[1-f\left(\varepsilon+\xi^{\prime}\right)\right]=f_{\mathrm{B}}\left(-\xi^{\prime}\right)\left[f\left(\varepsilon+\xi^{\prime}\right)-f(\varepsilon)\right],
$$

Eq. (A1) can be rewritten as a sum of integrals of the type $\int d \varepsilon f(\varepsilon) /(\varepsilon-\xi)^{n}(n=1,2, \ldots)$. In principal, it is necessary to determine the integrals for $n=1$, whereas the ones for $n>1$ can be found using the expression

$$
\int d \varepsilon \frac{f(\varepsilon)}{(\varepsilon-\xi)^{n+1}}=\frac{1}{n !} \frac{d^{(n)}}{d^{(n)} \xi} \int d \varepsilon \frac{f(\varepsilon)}{\varepsilon-\xi} .
$$


Below we demonstrate the calculation of one of the summands, namely

$$
J=\int d \varepsilon \frac{f(\varepsilon)}{\varepsilon+\mu_{\mathrm{L}}-\varepsilon_{\uparrow}} .
$$

To calculate this integral we use the Lorentzian cutoff of the form $g_{\alpha}(\varepsilon)=W^{2} /\left[\left(\varepsilon-\mu_{\alpha}\right)^{2}+W^{2}\right]$, with $W$ being the cutoff parameter. Thus, Eq. (A3) can be expressed as

$$
\begin{aligned}
J= & \frac{W}{2 i} \int d \varepsilon \frac{f(\varepsilon)}{\left(\varepsilon+\mu_{\mathrm{L}}-\varepsilon_{\uparrow}\right)\left(\varepsilon-\mu_{\mathrm{L}}-i W\right)} \\
& -\frac{W}{2 i} \int d \varepsilon \frac{f(\varepsilon)}{\left(\varepsilon+\mu_{\mathrm{L}}-\varepsilon_{\uparrow}\right)\left(\varepsilon-\mu_{\mathrm{L}}+i W\right)} .
\end{aligned}
$$

The first (second) integral in the above formula has poles at $\varepsilon=\varepsilon_{\uparrow}-\mu_{\mathrm{L}}, \varepsilon=\mu_{\mathrm{L}}+(-) i W$, and $\varepsilon=i(2 m+1) \pi$, with $m=0,1,2, \ldots$ However, because we are interested in the deep Coulomb blockade regime (where the second-order processes dominate), it is justifiable to assume $\varepsilon \ll \varepsilon_{\sigma}$ and this way neglect the contribution of the first pole. Then, by means of the contour integration and assuming $W$ to be the largest energy scale, one gets

$$
J=\operatorname{Re} \Psi\left(\frac{1}{2}+i \frac{\varepsilon_{\uparrow}-\mu_{\mathrm{L}}}{2 \pi k_{\mathrm{B}} T}\right)-\ln \left(\frac{W}{2 \pi k_{\mathrm{B}} T}\right) .
$$

As the single integral depends on the cutoff parameter, the total rate does not. The expressions depending on $W$ cancel in pairs, which can be simply seen from Eq. (A2). The other summands of Eq. (A1) can be found in a similar way with the aid of the above mentioned identities.

Another way to calculate the cotunneling rates is to make use of the assumption $\varepsilon \ll \varepsilon_{\sigma}$ and approximate the resolvents of Eq. (A1) by $1 /\left(\varepsilon+\mu_{\mathrm{L}}-\varepsilon_{\uparrow}\right) \approx 1 /\left(\mu_{\mathrm{L}}-\varepsilon_{\uparrow}\right)$. As a consequence, one arrives at the expressions of type $\int d \varepsilon f(\varepsilon)\left[1-f\left(\varepsilon+\xi^{\prime}\right)\right]$, which can be easily calculated 25]. The latter method is equivalent to the former one if one expands the digamma functions and neglects the higher-order corrections in $x / y$, with $x=|e V|, k_{\mathrm{B}} T$ and $y=\varepsilon_{\sigma}, \varepsilon_{\sigma}+U$. The advantage of using the first way of calculating the rates is that the higher-order corrections in temperature are properly described.

\section{References}

1. L. P. Kouwenhoven, C. M. Marcus, P. L. McEuen, S. Tarucha, R. M. Westervelt, and N. S. Wingreen, in Proceedings of the NATO Advanced Study Institute on Mesoscopic Electron Transport, Eds. L. L. Sohn, L. P. Kouwenhoven, and G. Schön (Kluwer Series E345, 1997).

2. L. Kouwenhoven and C. Marcus, Physics World, June 1998, p.35.

3. M. Jullière, Phys. Lett A 54, 225 (1975).

4. J. Barnaś and A. Fert, Phys. Rev. Lett. 80, 1058 (1998); Europhys. Lett. 24, 85 (1998).

5. A. Brataas, Yu. V. Nazarov, J. Inoue and G. E. W. Bauer, European Phys. Journ. B 9, 421 (1999); Phys. Rev. B 59, 93 (1999).
6. K. Majumdar and S. Hershfield, Phys. Rev. B 57, 11521 (1998).

7. I. Weymann and J. Barnaś, Phys. Status Solidi 236, 651 (2003).

8. K. Yakushiji, S. Mitani, K. Takanashi, and H. Fujimori, J. Appl. Phys. 91, 7038 (2002).

9. F. Ernult, K. Yamane, S. Mitani, K. Yakushiji, K. Takanashi, Y. K. Takahashi, K. Hono J. Appl. Phys. 84, 3106 (2004).

10. B. R. Bułka, Phys. Rev. B 62, 3186 (2000).

11. W. Rudziński and J. Barnaś, Phys. Rev. B 69, 085318 (2001)

12. I. Weymann, J. Barnaś, J. König, J. Martinek, and G. Schön, cond-mat/0412434

13. R. Świrkowicz, J. Barnaś, and M. Wilczyński, J. Phys.: Condens. Matter 14, 2011 (2002).

14. Y. Utsumi, J. Martinek, G. Schön, H. Imamura, S. Maekawa, cond-mat/0501172

15. J. Martinek, Y. Utsumi, H. Imamura, J. Barnaś, S. Maekawa, J. König, and G. Schön, Phys. Rev. Lett. 91, 127203 (2003).

16. R. Lopez and D. Sanchez, Phys. Rev. Lett. 90, 116602 (2003).

17. N. Sergueev, Qing-feng Sun, Hong Guo, B. G. Wang, and Jian Wang, Phys. Rev. B 65, 165303 (2002).

18. J. König and J. Martinek, Phys. Rev. Lett. 90, 166602 (2003).

19. M. Braun, J. König, J. Martinek, Phys. Rev. B 70, 195345 (2004).

20. W. Rudziński, J. Barnaś, R. Świrkowicz, and M. Wilczyński, cond-mat/0409386

21. J. N. Pedersen, J.Q. Thomassen, and K. Flensberg, cond-mat/0412145

22. J. Fransson, cond-mat/0502288

23. J. Fransson, O. Eriksson, and I. Sandalov, Phys. Rev. Lett. 88, 226601 (2002).

24. This follows directly from the general kinetic equation in the Liouville space for the density matrix elements derived within the real-time diagrammatic technique [19].

25. D. V. Averin and Yu. V. Nazarov, Phys. Rev. Lett. 65, 2446 (1990); in Single Charge Tunneling, edited by $\mathrm{H}$. Grabert and M. Devoret (Plenum, New York 1992).

26. K. Kang and B. I. Min, Phys. Rev. B 55, 15412 (1997).

27. J. König and Y. Gefen, Phys. Rev. Lett. 86, 3855 (2001).

28. A. Kogan, S. Amasha, D. Goldhaber-Gordon, G. Granger, M. A. Kastner, and H. Shtrikman, Phys. Rev. Lett. 93, 166602 (2004). 
